\title{
ON FINITE DIMENSIONAL APPROXIMATIONS OF MAPPINGS IN BANACH SPACES ${ }^{1}$
}

\author{
JESt'S GIL DE LAMADRID
}

1. Introduction. In this note we discuss three somewhat disconnected results. However, they are related by the fact that they have to do with pointwise approximations of mappings by finite dimensional mappings and that they imply the uniform approximation of certain compact mappings by finite dimensional mappings. $\$ 2$ has to do with a question raised by Day [2] concerning a characterization of Hilbert space. $\$ 3$ presents an alternate proof of the result of Grothendieck [4] which states that the space $C(X)$ of all continuous bounded functions on a topological space $X$ satisfies the condition of metric approximation. $\$ 4$ presents a necessary and sufficient condition for the compactness of the derivative mapping of a real valued function on a Banach space.

Throughout this note $E$ will stand for a Banach space with norm \|\| . All topological concepts involving a Banach space refer to its norm topology.

2. Characterization of inner product topologies. In $[2$, p. 120] the question is asked if there exists a Banach space $E$, not homeomorphically isomorphic to a Hilbert space with the following property

$D$ : There is a number $M>0$ so that for every closed vector subspace $F \subset E$ there is a projection $P_{F}: E \rightarrow F$ with $\left\|P_{P}\right\|<M$.

Theorem 1, an easy consequence of Kakutani's well-known theorem [5, p. 94], gives a characterization of inner product spaces in terms of a property which is slightly stronger than $\mathrm{D}$. The property is

$\mathrm{D}^{\prime}$ : There is a number $M>0$ so that for every closed vector subspace $F \subset E$ there is a projection $P_{B}: E \rightarrow F$ so that the multiplicative semigroup $S$ generated by $\left\{P_{\boldsymbol{p}}\right\}_{F_{\mathrm{C}} E}$ is bounded by $M$.

THEOREM 1. The space $E$ has property $\mathrm{D}^{\prime}$ if and only if it is homeomorphically isomorphic to a Hilbert space.

Proof. "If." If $E$ is homeomorphically isomorphic to a Hilbert space, its norm \|\| can be replaced by an inner product norm \|\|$^{\prime}$ preserving the topology of $E$. For each closed vector subspace $F \subset E$, we let $P_{F}$ be the orthogonal projection onto $F$. Then the semigroup

Received by the editors January 19, 1961.

${ }^{1}$ Research supported by NSF grant NSFG 14137. 
$S$ generated by $\left\{P_{\boldsymbol{P}}\right\}_{\boldsymbol{P}}$ is bounded by 1 , with respect to \|\|$^{\prime}$ and by some number $M$ with respect to \|\| .

"Only if." Suppose $S$ is uniformly bounded with respect to \|\| . For $x \in E$, let $\|x\|^{\prime}=\sup _{A \in S}\|A x\|$. Clearly \|\|$^{\prime}$ is a norm $\geqq\|\|$. It is actually equivalent to \|\| , by uniform boundedness of $S$. We show $\left\|P_{P}\right\|^{\prime}=1$, for every $F$. For every $x \in E,\left\|P_{P} x\right\|^{\prime}=\sup _{A \in S}\left\|A P_{P} x\right\|$ $\leqq \sup _{B \in S}\|B x\|=\|x\|^{\prime}$. That \|\|$^{\prime}$ is an inner product norm now follows from Kakutani's well-known theorem [5, Theorem 2, p. 94].

We remark briefly that if $E$ has property $\mathrm{D}$, then it satisfies the condition of approximation of Grothendieck [4, p. 167, Definition 9]. Since the identity transformation $I$ is a pointwise limit point of the set $\left\{P_{F}\right\}_{F}$ which is uniformly bounded, a familiar argument yields that $I$ is a limit point of $\left\{P_{F}\right\}_{F}$ in the topology of uniform convergence over compact sets.

3. The space of continuous functions. Grothendieck has shown $[4, \mathrm{p} .185]$ that the space $C(X)$ of all continuous bounded functions over a topological space $X$ with the supremum norm satisfies his condition of metric approximation. This condition states that the identity transformation can be approximated pointwise by finite dimensional linear transformations of norm less than or equal to 1 . His proof is based on the same property of approximation which he establishes for the dual of $C(X)$, which can be interpreted as a space $L_{1}(\mu)$ for a suitable measure $\mu$. The proof is measure theoretic. Since this theorem is rather important it might be of interest to have a completely different more direct proof. This section is devoted to presenting such a proof, based on a method of Leray and Schauder [6], utilized by Rothe [8] for a purpose similar to the present one. Without loss of generality we may assume that $X$ is compact and Hausdorff.

We assume the space $X$ embedded in $[C(X)]^{\prime}$ and treat its points as functionals. By $[7$, Theorem 2, p. 500], for every weak* neighborhood $U$ of $0 \in[C(X)]^{\prime}$, there is a finite-dimensional weakly* continuous mapping $\phi_{U}: X \rightarrow[C(X)]^{\prime}$ (linearity has no meaning for $\phi_{U}$ ) so that $\phi_{U} x-x \in U$ for every $x \in X$. Define, for $f \in C(X),\left(T_{U} f\right)(x)$ $=\left\langle f, \phi_{U}(x)\right\rangle . T_{U} f$ is clearly in $C(X)$, and $T_{U}$ is linear. In the remainder of our proof we must compute with the formula for $\phi_{U}$, but skip some of the reasoning. See [7] for details and [8, pp. 526-527] for a particularly instructive discussion. $U$ may be assumed to be given by a finite set $\left\{f_{i}\right\}_{i}^{m} C C(X)$ and $\epsilon>0$ via $\left|\left\langle f_{i}, f^{\prime}\right\rangle\right|\left\langle\epsilon, f^{\prime} \in[C(X)]^{\prime}\right.$. Then $\phi_{U}$ may be assumed of the form 


$$
\phi_{U}(x)=\sum_{k=1}^{n} \mu_{k}(x) x_{k} / \mu(x)
$$

for suitably chosen points $x_{k} \in X$,

$$
\mu_{k}(x)=\left(\epsilon-\bigvee_{i=1}^{m}\left|f_{i}(x)-f_{i}\left(x_{k}\right)\right|\right) \bigvee 0,
$$

$V$ standing for $\max$ and

$$
\mu(x)=\sum_{k=1}^{n} \mu_{k}(x)>0
$$

for $x \in X$. Each $\mu_{k}$ is continuous in $x$. $T_{U}$ is clearly finite dimensional, its range spanned by the functions $\mu_{k} / \mu$. Also $\left|\left(T_{U} f\right)(x)\right| \leqq \max _{k}\left|f\left(x_{k}\right)\right|$ $=\|f\|$. Hence $\left\|T_{U}\right\| \leqq 1$. Finally, given $f \in C(X), \epsilon>0$ and $U$ defined by $f$ and $\epsilon$,

$$
\left|f(x)-\left(T_{U} f\right)(x)\right|=\left[\sum_{k=1}^{n} \mu_{k}(x)\left|f(x)-f\left(x_{k}\right)\right|\right] / \mu(x)<\epsilon .
$$

Hence $f=\lim _{U} T_{U} f$. This completes our proof.

4. Compact derivatives. The approximations in this section are connected with a condition equivalent to the compactness of the derivative, or gradient mapping $f^{\prime}$ of a Gâteaux differentiable (see $[3$, p. 417]) real valued function $f$ defined on an open subset $D$ of $E$. A condition on $f$ was introduced by Rothe [8, p. 428, Theorem 3.3] who proved it necessary, and, in the case of Hilbert spaces, sufficient for the compactness of $f^{\prime}$. In a paper recently submitted to these Proceedings by T. Andô [1], who communicated his results to me by letter, the condition of Rothe has been proved sufficient for perfectly general Banach spaces. The condition presented here is closely related to the earlier one and its sufficiency can be proved for bounded $f$ as a corollary to the result of Andô. However, the proof of sufficiency given below, conceived independently, does not demand that $f$ be bounded and is based on an idea that does not appear in earlier works.

TheOREM 2. Let $D$ be an open subset of $E$ and $f$ a real valued function defined on $D$ which has a continuous Gateaux derivative. Then its derivative $f^{\prime}: D \rightarrow E^{\prime}$ is compact if and only if $f$ satisfies the following condition:

For every $\epsilon>0$ there exist a finite number of functionals $\left\{x_{i}^{\prime}\right\}_{i}$ so

* that to every $x \in D$ one can associate an $i$ and $a \delta_{x}>0$ for which $h \in E,\|h\|<\delta_{x}$ and $\left|\left\langle h, x_{i}\right\rangle\right|<\epsilon\|h\|$ imply $|f(x+h)-f(x)|<\epsilon\|h\|$. 
LEMMA 1. Under the hypothesis of Theorem 2, condition * of that theorem is equivalent to the following condition on $f^{\prime}$ :

For every $\epsilon>0$ there exist a finite number of functionals $\left\{x_{i}^{\prime}\right\}_{i}$ so ** that for every $x \in D$, there is an $i$ so that $h \in E$ and $\left|\left\langle h, x_{i}^{\prime}\right\rangle\right|\langle\epsilon\|h\|$ imply

$$
\left|\left\langle h, \jmath^{\prime}(x)\right\rangle\right|<\epsilon\|h\| \text {. }
$$

Proof. Condition * implies Condition ${ }^{* *}$. Let $\epsilon>0$, and $\left\{x_{i}^{\prime}\right\}_{i}$ be the set of functionals obtained from Condition * for $\epsilon / 2$. Then the set $\left\{2 x_{i}^{\prime}\right\}_{i}$ and $\epsilon$ are suitable for Condition ${ }^{* *}$, for, if $x \in D$ and $i$ is so that $\left|\left\langle h, x_{i}^{\prime}\right\rangle\right|<(\epsilon / 2)\|h\|$ implies $|f(x+h)-f(x)|<(\epsilon / 2)\|h\|$, then, for small enough $t$, in the inequality

$$
\begin{aligned}
\left|\left\langle h, f^{\prime}(x)\right\rangle\right| \leqq & \left|\left\langle h, f^{\prime}(x)\right\rangle-[f(x+t h)-f(x)] / t\right| \\
& +|[f(x+t h)-f(x)] / t|
\end{aligned}
$$

each of the two terms on the right is smaller than $(\epsilon / 2)\|h\|$.

Condition ${ }^{* *}$ implies Condition ${ }^{*}$. This is a similar argument, which we merely outline. From

$$
f(x+h)-f(x)=\int_{0}^{1}\left\langle h, f^{\prime}(x+t h)\right\rangle d t
$$

we see that if $\left|\left\langle h, f^{\prime}(x)\right\rangle\right|$ is made less than $(\epsilon / 2)\|h\|$ in the manner indicated in Condition ${ }^{* *}$, the intgral in (2) can be made smaller than $\epsilon\|h\|$, for $\|h\|$ small enough, by continuity of $f^{\prime}$.

In view of Lemma 1 it suffices to prove that Condition ${ }^{* *}$ is equivalent to compactness of $f^{\prime}$.

Before embarking on such a proof we remark that, for $x_{0}^{\prime} \in E^{\prime}$, $x_{0}^{\prime} \neq 0, x_{0} \in E,\left\|x_{0}\right\|=1$ and $\left\langle x_{0}, x_{0}^{\prime}\right\rangle$ within $\epsilon\left\|x_{0}^{\prime}\right\|$ of $\left\|x_{0}^{\prime}\right\|$, the projection $P_{x_{00^{\prime}}}: E \rightarrow E$, defined by

$$
P_{x_{0}} x=\frac{\left\langle x, x_{0}^{\prime}\right\rangle}{\left\langle x_{0}, x_{0}^{\prime}\right\rangle} x_{0},
$$

is such that $\left\|P_{x_{0}}\right\|<1 /(1-\epsilon)$, for

$$
\frac{\left|\left\langle x, x_{0}^{\prime}\right\rangle\right|}{\left|\left\langle x_{0}, x_{0}^{\prime}\right\rangle\right|}<\frac{\|x\|\left\|x_{0}^{\prime}\right\|}{\left\|x_{0}^{\prime}\right\|(1-\epsilon)}=\frac{\|x\|}{1-\epsilon} .
$$

The projection $P_{x_{0^{\prime}}}^{\prime}$ defined by

$$
P_{x_{0}{ }^{\prime}}^{\prime} x^{\prime}=\frac{\left\langle x_{0}, x_{0}^{\prime}\right\rangle}{\left\langle x_{0}, x_{0}^{\prime}\right\rangle} x_{0}^{\prime} .
$$


is the transpose of $P_{x_{0^{\prime}}}$, and, therefore has the same norm. Hence $\epsilon$ can be chosen so that $\left\|P_{x_{0} 0^{\prime}}\right\|=\left\|P_{x_{0} 0^{\prime}}^{\prime}\right\|<2$. Henceforth we shall assume $\epsilon$ so chosen. Suppose now that $x^{\prime} \in E^{\prime}$ and that $\left|\left\langle h, x_{0}^{\prime}\right\rangle\right|\langle\epsilon\|h\|$ implies $\left|\left\langle h, x^{\prime}\right\rangle\right|<\epsilon\|h\|$. Then $\left\|x^{\prime}-P_{x_{0}}^{\prime} x^{\prime}\right\|<3 \epsilon$, for, if $x \in E,\left|\left\langle x, x^{\prime}-P_{x_{0}}^{\prime} x^{\prime}\right\rangle\right|$ $=\left\langle x-P_{x_{0}, x}, x^{\prime}\right\rangle$. Since $\left|\left\langle x-P_{x_{0}} x, x_{0}^{\prime}\right\rangle\right|=0<\epsilon\left\|x-P_{x_{0} 0^{\prime}} x\right\|$, $\left|\left\langle x-P_{x_{0}{ }^{\prime}} x, x^{\prime}\right\rangle\right|<\epsilon\left\|x-P_{x_{0}, x}\right\| \leqq 3 \epsilon\|x\|$.

Proof of Theorem 2. The proof that compactness of $f^{\prime}$ implies ** is very similar to a special case of the proof of Theorem 10 of [3] and will be omitted. We show that ${ }^{* *}$ implies compactness of $f^{\prime}$. Let ${ }^{* *}$ be satisfied. We show that $f^{\prime}(D)$ is precompact. Let $\epsilon>0$ and $\left\{x_{i}^{\prime}\right\}_{i}^{n}$ be the finite set provided by ${ }^{* *}$ for $\epsilon$, and

$$
A_{i}=\left\{x^{\prime}\left|x^{\prime} \in f^{\prime}(D),\right|\left\langle h, x_{i}^{\prime}\right\rangle \mid<\epsilon\|h\| \text { implies }\left|\left\langle h, x^{\prime}\right\rangle\right|<\epsilon\|h\|\right\} .
$$

Then $f^{\prime}(D)=\bigcup_{i=1}^{n} A_{i}$ and each $P_{x_{i}{ }^{\prime}}^{\prime}\left(A_{i}\right)$ is a bounded one-dimensional set, for there is $h_{i} \in E$ so that $0<\left\langle h_{i}, x_{i}^{\prime}\right\rangle\left\langle\epsilon\left\|h_{i}\right\|_{\text {. Then, for every }}\right.$ $x^{\prime} \in A_{i},\left|\left\langle h_{i}, P_{x_{i}}^{\prime} x^{\prime}\right\rangle\right| \leqq\left|\left\langle h_{i}, P_{x_{i}}^{\prime} x^{\prime}-x^{\prime}\right\rangle\right|+\left|\left\langle h_{i}, x^{\prime}\right\rangle\right|<4 \epsilon\left\|h_{i}\right\|$. On the other hand $P_{x_{i}}^{\prime} x^{\prime}=\lambda x_{i}^{\prime}$ for suitable $\lambda$. Then

$$
\begin{aligned}
\left\|P_{x_{i}^{\prime}}^{\prime} x^{\prime}\right\| & =|\lambda|\left\|x_{i}^{\prime}\right\|=\frac{|\lambda|\left\langle h_{i}, x_{i}^{\prime}\right\rangle}{\left\langle h_{i}, x_{i}^{\prime}\right\rangle}\left\|x_{i}^{\prime}\right\| \\
& =\left|\left\langle h_{i}, P_{x_{i}}^{\prime} x^{\prime}\right\rangle\right| \frac{\left\|x_{i}^{\prime}\right\|}{\left\langle h_{i}, x_{i}^{\prime}\right\rangle}=\frac{4 \epsilon\left\|h_{i}\right\|\left\|x_{i}\right\|}{\left\langle h_{i}, x_{i}^{\prime}\right\rangle} .
\end{aligned}
$$

Hence $P_{x_{i}{ }^{\prime}}^{\prime}\left(A_{i}\right)$ is bounded and one-dimensional. So it is precompact. Therefore it contains a set ${ }_{1} x_{i}^{\prime},{ }_{2} x_{i}^{\prime}, \cdots,{ }_{s_{i}} x_{i}^{\prime}$ so that for every $x^{\prime} \in A_{i}$, $\left\|P_{x_{i}}^{\prime} x^{\prime}-{ }_{k} x_{i}^{\prime}\right\|$ for some $k$. Hence, for every $x^{\prime} \in f^{\prime}(D)$

$$
\left\|x^{\prime}-{ }_{k} x_{i}^{\prime}\right\| \leqq\left\|x^{\prime}-P^{\prime} x_{i_{i}} x^{\prime}\right\|+\left\|P_{x_{i}}^{\prime} x^{\prime}-{ }_{k} x_{i}^{\prime}\right\|<5 \epsilon
$$

for some $i$ and some $k, 1 \leqq k \leqq s_{i}$. From here we conclude that $f^{\prime}(D)$ is precompact.

\section{BIBLIOGRAPHY}

1. T. Andô, On gradient mappings in Banach spaces, Proc. Amer. Math. Soc. 12 (1961), 297-299.

2. M. M. Day, Normed linear spaces, Ergebnisse der Math. und ihre Grenzgebiete, (N. F.) Heft 28, 1958.

3. J. Gil de Lamadrid, Topology of mappings and differentiation processes, Illinois J. Math. 3 (1959), 408-420.

4. A. Grothendieck, Produits tensoriels topologiques et espaces nucleaires, Mem. Amer. Math. Soc. No. 16 (1955) 140 pp.

5. S. Kakutani. Some characterizations of euclidean spaces, Jap. J. Math. 16 (1939), 93-97. 
6. J. Leray and J. Schauder, Topologie et equations fonctionnelles, Ann. Sci. Ecole Norm. Sup. (3) 5 (1934), 45-78.

7. M. Nagumo, Degree of mapping in convex topological spaces, Amer. J. Math. 73 (1951), 497-511.

8. E. H. Rothe, Gradient mappings in Banach spaces, Duke Math. J. 15 (1948), $421-431$.

UNIVERSITY OF MiNNESOTA

\title{
COEFFICIENT ESTIMATES FOR UNIVALENT FUNCTIONS
}

\author{
P. L. DUREN
}

Let

$$
f(z)=z+b_{1} / z+b_{2} / z^{2}+\cdots
$$

be analytic and univalent in $|z|>1$. It is an immediate consequence of the area theorem that $\left|b_{1}\right| \leqq 1$. In 1938 Schiffer [5], using a variational method, and Golusin [2] independently derived the sharp inequality $\left|b_{2}\right| \leqq 2 / 3$. It was then conjectured that $\left|b_{n}\right| \leqq 2 /(n+1)$, but this proved to be false without additional hypotheses. Indeed, Garabedian and Schiffer [1] found the true bound for $\left|b_{3}\right|$ to be $1 / 2+e^{-6}$. A theorem of Golusin [2], however, asserts the following:

THEOREM. Let $f(z)$ be analytic and univalent in $|z|>1$ and have the expansion (1). For some $N \geqq 1$, let $b_{1}=b_{2}=\cdots=b_{N-1}=0$. Then $\left|b_{n}\right| \leqq 2 /(n+1)$ for $n=N, N+1, \cdots, 2 N$. (In particular, $\left|b_{2}\right| \leqq 2 / 3$.)

Jenkins [4] has recently obtained this result by means of his General Coefficient Theorem. It is the purpose of the present note to give a simple proof. Clearly, it suffices to show $\left|b_{2 N-1}\right| \leqq 1 / N$ and $\left|b_{2 N}\right| \leqq 2 /(2 N+1)$.

The $n$th Faber polynomial $F_{n}(w)$ associated with the function (1) is defined by

$$
F_{n}[f(z)]=z^{n}+\sum_{m=1}^{\infty} \beta_{n m} z^{-m}
$$

The following inequalities, due to Grunsky [3], constitute an elementary generalization of the area theorem: 1960.

Presented to the Society, August 29, 1961; received by the editors December 17, 